\title{
On the spectral resolution of the MILES stellar library (Research Note)
}

\author{
A. Beifiori, C. Maraston, D. Thomas, and J. Johansson
}

\begin{abstract}
Institute of Cosmology and Gravitation, University of Portsmouth, Dennis Sciama Bldg, Burnaby Road, Portsmouth, PO1 3FX, UK SEPNET, South East Physics Network, UK

e-mail: alessandra.beifiori@port.ac.uk
\end{abstract}

Received 14 December 2010 / Accepted 16 May 2011

\begin{abstract}
Context. Empirical stellar libraries are extensively used to extract stellar kinematics in galaxies and to build stellar population models. An accurate knowledge of the spectral resolution of these libraries is critical for avoiding propagation errors and uncertain estimates of the intrinsic stellar velocity dispersion of stellar systems, such as galaxies and star clusters.

Aims. In this research note we re-assess the spectral resolution of the MILES stellar library and of the stellar population models based on it. This exercise was performed, because of a recent controversy over the exact MILES resolution.

Methods. We performed our test by comparing MILES stellar spectra with three different sets of higher resolution templates, one fully theoretical - the MARCS library - and two empirical ones, namely the Indo-US and ELODIE v3.1 libraries. The theoretical template has a well-defined, very high $(R=20000)$ resolution, so errors in this theoretical value do not affect our conclusions. Our approach based on the MARCS library was also crucial for constraining the resolution of the two empirical templates.

Results. We find that the MILES resolution is coarser than previously stated. We derive a new spectral resolution of $2.54 \AA F W H M$, instead of the nominal $2.3 \AA$. The reason for this difference is the previous overestimation of the resolution for the Indo-US library, which was used to estimate the MILES one. For the Indo-US library we obtain a new value of $1.35 \AA$ FWHM. Most important, the results derived from the MARCS and ELODIE libraries are in very good agreement.

Conclusions. These results are important for users of the MILES spectra library and for further development of stellar population models aimed at obtaining accurate stellar kinematics and spectroscopic properties of galaxies.
\end{abstract}

Key words. techniques: spectroscopic - stars: kinematics and dynamics - galaxies: kinematics and dynamics

\section{Introduction}

Stellar libraries are a crucial ingredient in stellar population models that predict the spectral energy distributions of stellar populations (e.g., Worthey 1994; Fioc \& Rocca-Volmerange 1997; Bruzual \& Charlot 1993; Maraston 1998, 2005; Maraston et al. 2009a). The latter are a key tool for analysing unresolved stellar populations such as galaxies and extra-galactic star clusters. The spectral resolution of a stellar population model is determined by the spectral resolution of the input stellar library, which can be either theoretical or empirical. As theoretical model atmosphere calculations are known to suffer from incomplete line lists and continuum uncertainties (Korn et al. 2005; Thomas et al. 2011; Maraston \& Strömbäck 2011), empirical stellar libraries have become a complementary and widely used option for calculating stellar population models (Vazdekis 1999; Vazdekis et al. 2010; Bruzual \& Charlot 2003; Le Borgne et al. 2004; Schiavon 2007; Maraston et al. 2009b; Maraston \& Strömbäck 2011). The spectral resolution of empirical libraries is fixed by the instrumental resolution of the observations. An accurate assessment of the underlying spectral resolution of both the input library and the final stellar population model is essential for proper use of such models.

Several new empirical stellar libraries have been published in recent years, e.g., ELODIE (Prugniel \& Soubiran 2001), STELIB (Le Borgne et al. 2003), and MILES (SánchezBlázquez et al. 2006). The new stellar population models of Maraston \& Strömbäck (2011) include all three libraries, while the new model of Lick absorption-line indices by Thomas et al. (2011) and Johansson et al. (2010) is based on MILES. In both Maraston \& Strömbäck (2011) and Thomas et al. (2011) it is pointed out that the stellar population spectral resolution of MILES appears to be somewhat coarser than generally assumed. It has been found that velocity dispersions of galaxies from the Sloan Digital Sky Survey (SDSS) data base (York et al. 2000) derived using the MILES-based templates of Maraston \& Strömbäck (2011) agree well with the values from the SDSSMPA/JHU data base (Kauffmann et al. 2003; Tremonti et al. 2004). This indicates that stellar population models based on MILES are close to the SDSS spectral resolution $(R \sim 1800$ 2000 at $5000 \AA$ ), so are somewhat coarser than the $2.3 \AA F W H M$ stated in Sánchez-Blázquez et al. (2006) and Vazdekis et al. (2010).

The aim of this research note is to assess the spectral resolution of the MILES stellar library through direct comparison with various other empirical and theoretical stellar libraries at higher spectral resolution. Since a stellar population model may further dilute the nominal spectral resolution of the input library owing to uncertainties in the radial velocities of input stars (MacArthur et al. 2009), we additionally assess the spectral resolution of the MILES-based Maraston \& Strömbäck (2011) model.

\section{Template stellar libraries}

The MILES library (Sánchez-Blázquez et al. 2006) comprises 985 stars for a wide range of evolutionary stages and 
metallicities. It covers a wavelength range from 3525-7500 at a nominal spectral resolution of $2.3 \AA F W H M$. This value has been determined in Sánchez-Blázquez et al. (2006) through the comparison with the higher resolution empirical library Indo-US (Valdes et al. 2004).

The result from this evaluation is only as robust as the spectral resolution of the adopted high-resolution library. Therefore, in this research note we select three different stellar libraries, both theoretical and empirical, with spectral resolutions higher than the MILES one, with the aim at checking the robustness of this value.

The adopted libraries are Indo-US, MARCS and ELODIE v3.1. The key to our analysis is that MARCS is a theoretical library therefore with a well-defined spectral resolution. We summarise below the main characteristics of these libraries.

- Indo-US empirical library: consists of 1273 stars spectra covering the wavelength range between $3460-9464 \AA$ with a resolution of $\sim 1.2 \AA F W H M$ (Valdes et al. 2004). Although the resolution of Indo-US varies throughout the library (due to the long observation period with slightly different set up, Valdes et al. 2004; Wu et al. 2011) and therefore is not suitable for measurements of line broadening, we used this template for consistency with the method of Sánchez-Blázquez et al. (2006). The library has a broad coverage of the stellar atmospheric parameters effective temperature, surface gravity and metallicity.

- MARCS theoretical library: contains high-resolution $(R=$ $20000)$ theoretical spectra and covers a large spectral range from $1300 \AA$ to $20 \mu \mathrm{m}$ Gustafsson et al. (2008). Effective temperatures range between $2500-8000 \mathrm{~K}$ (at solar metallicity), surface gravities between -1.0 and 5.5 and metallicities between -5.0 and +1.0 . Although the resolution of a theoretical library is well defined and not affected by observational issues, the line broadening is maybe affected by line list incompleteness, uncertainties in the model atmosphere models, and oscillator strengths (Kurucz 1979; Gustafsson et al. 2008).

- ELODIE v.3.1 empirical library: this library is an updated release of the original ELODIE (Prugniel \& Soubiran 2001; Prugniel et al. 2007). It includes 1962 spectra of 1388 stars. ELODIE v3.1 covers a wavelength range between 3900$6800 \AA$ and provides a large coverage of atmospheric parameters. It is given at two resolutions, $R \sim 42000$ and $R \sim$ 10000 . We decided to select the $R \sim 42000$ (LH_ELODIE), which is more adequate for a robust test of the MILES library resolution. Any error or variation of the resolution with wavelength (Baranne et al. 1996) would not appreciably affect our results (the variation is two order of magnitude lower than the typical measurement error) because of the large difference between the resolution of the two libraries. The constant $R$ translates in a varying $F W H M$ line broadening with wavelength. We accounted for this by estimating the ELODIE's FWHM for each bin prior to estimating the absolute broadening.

\section{Method}

The spectral resolution is characterised by the line broadening given by the intrinsic resolution of a spectrograph and the slit function (for a detailed description see Koleva et al. 2008, 2009; $\mathrm{Wu}$ et al. 2011). The line broadening can change among the stars of an empirical library because it also depends on the stellar light profile and on the specific observing conditions. Additional uncertainties can be added during data reduction and wavelength calibration. Therefore it is very important to assess this variation for the final empirical library before its use for population modelling or stellar kinematics.

In order to extract the resolution of the MILES spectra we follow a similar procedure as in Sánchez-Blázquez et al. (2006). We first divide each spectrum in 11 regions equally spaced in $\log$ space. This enable us to assess the dependence of the resolution on wavelength. We parameterised the spectral resolution with the FWHM measured assuming a Gaussian shape for the instrumental broadening of the spectral lines. We then derive the instrumental broadening of each MILES star with respect to a library of templates at higher resolution (Indo-US, MARCS, ELODIE v3.1) by using the Penalized Pixel-fitting method (pPXF) of Cappellari \& Emsellem (2004) and taking into account the resolution of each template. pPXF performs the fitting in the pixel scale between an observed spectrum and a linear positive combination of different templates.

We fitted the logarithmically rebinned spectra with a Gaussian function ${ }^{1}$ letting both the first and the second moment, velocity and sigma, free, as well as the weights free for each independent segment. We tested that by applying one single optimal template derived by fitting the whole spectrum to each bin, we derived a fully consistent resolution. In each segment we first determined the relative line broadening of the MILES spectra with respect to the set of templates at higher resolution and then we added in quadrature the template line broadening under analysis. The code pPXF also allows the use of additive and multiplicative Legendre polynomials for adjusting the continuum shape of the template to the one of the spectrum to be analysed. For our analysis we used a fourth order additive polynomial. Tests revealed this was the stablest choice, although multiplicative polynomials or no polynomials would give consistent results within $1 \sigma$ error.

Our results are stable against all tests we made meaning that the free parameters in our fit are independent enough and do not require additional regularisation.

The comparison between the results of two empirical libraries and a theoretical library indicates not only on the resolution of MILES but also on the actual resolution of the empirical templates we use. Hence, by using the same method, we additionally estimate the actual FWHM of the Indo-US library using as templates MARCS and ELODIE v3.1 libraries. Note that the very high resolution of our templates - ELODIE v3.1 $(R=42000)$ and MARCS $(R=20000)$ - make us confident in our results since any error in the nominal resolution has very little impact on the final derived value.

After obtaining the values of the broadening for each MILES star we derived the median of FWHMs for avoiding being dominated by spurious values. Errors for each wavelength bin were estimated as the standard deviation of the FWHMs. This estimation is validated by the fact that the typical distribution of $F W H M$ is close to a Gaussian (see Fig. 1 for one example). In Fig. 2 we show the results for each wavelength bin and for each template library as described below.

With the same procedure we derived the resolution of the MILES-based SSP of Maraston \& Strömbäck (2011) by using a representative subsample of them covering the whole age range $-6.5 \mathrm{Myr}, 1 \mathrm{Gyr}$ and $10 \mathrm{Gyr}$. This is important as the fractional

\footnotetext{
1 Since we used a Gaussian function we did not use the ppxf's penalisation that can be applied to the Gauss-Hermite high order moments once the higher moments are unconstrained by the data.
} 


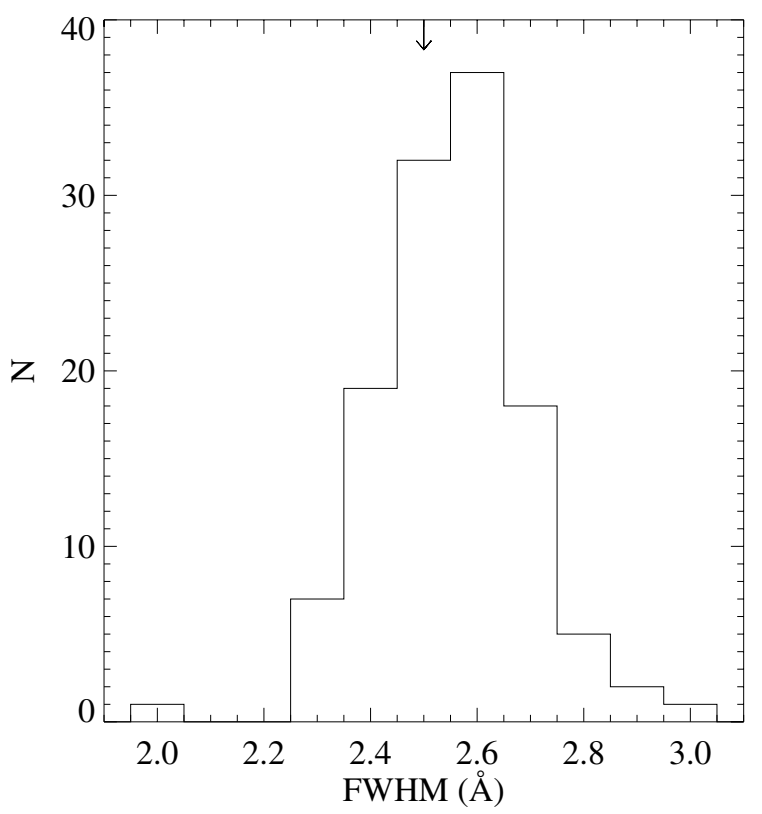

Fig. 1. Distribution of $F W H M$ for the 122 MILES solar metallicity stars fitted with the 653 ELODIE v3.1 solar metallicity spectra for the wavelength bin from $5160-5516 \AA$. The median of this distribution is marked by an arrow.

contribution of dwarfs and giants to the integrated stellar population spectrum changes with age. We use solar metallicity models with a Salpeter IMF.

\section{Results}

In the following we describe the results for each template separately. Note that in all cases we decided to remove the reddest wavelength interval (6937-7500 $⿱$ ) as we could not obtain a good fit to the spectra, possibly due to the lack to strong absorption features to constrain the fit.

\subsection{Template: Indo-US library at FWHM 1.2}

As a first test we followed the same procedure of SánchezBlázquez et al. (2006). We first fitted each 985 MILES spectra with a linear combination of Indo-US spectra (1274 templates). Then we applied the same technique to a subsample of stars chose among the MILES and Indo-US solar metallicity stars. This was done for testing possible bias due to the selection of a subsample of stars and also for enabling a comparison with the MILES-based stellar population models with solar metallicity. We choose all stars with metallicity in the range of $[\mathrm{Fe} / \mathrm{H}]=[\mathrm{Fe} / \mathrm{H}]_{\odot} \pm 0.05$ finding 122 and 146 stars, for MILES and Indo-US respectively. We did not find any bias between the initial sample and the final one, which excludes template mismatch and an insufficient number of templates. In the following we will use only the solar metallicity subsample. The results are shown in panel a) of Fig. 2 (see the distribution of blue and red point in Fig. 2 panel a) for differences between all MILES stars and the solar metallicity subsample). We also tested the instrumental broadening derived by fitting the 377 stars in common between the MILES and the Indo-US libraries, finding consistent results within the errors (orange points in panel a) of Fig. 2).

Results are consistent for most wavelength regions. We found a coarser resolution in the first bin ranging from
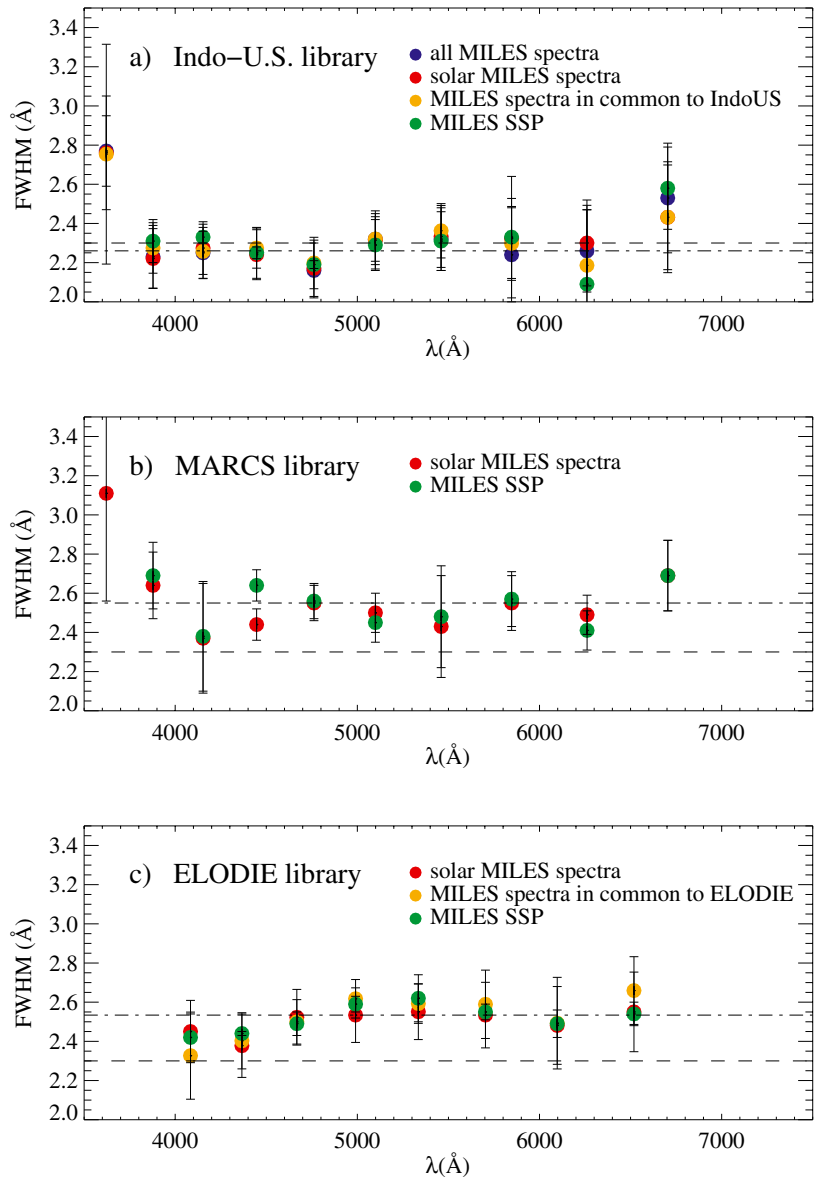

Fig. 2. $F W H M$ as a function of wavelength for the three different templates, panel a) Indo-US, panel b) MARCS, and panel c) ELODIE v.3.1 library. Different colours identify the MILES library (blue), the subsample of MILES stars with solar metallicity (red), the subsample of MILES stars in common with either Indo-US or ELODIE (orange), the MILES SSP from Maraston \& Strömbäck (2011) (green). The dashed line represents the nominal $F W H M=2.3 \AA$ (Sánchez-Blázquez et al. 2006), whereas the dotted-dashed line represents the median of the $F W H M$ obtained here. In the first wavelength bin of panel a) and b) the FWHM of SSP are not shown because out from the FWHM range (see Maraston \& Stromback for details).

3525-3748 $\AA$, possibly due to the higher residuals in the fit we found in this wavelength interval. For the stellar population models in particular, this first bin gives a relatively poor resolution of $4.31 \pm 0.03 \AA F W H M$, hence a significantly lower spectral resolution. This wavelength bin is therefore excluded from the analysis.

We also notice a difference between the resolution in the redder and bluer parts of the spectrum but with a variation that is negligible within the errors. The median FWHM is $2.26 \pm$ $0.08 \AA, 2.32 \pm 0.08 \AA$ and $2.30 \pm 0.09 \AA$ by using the whole Indo-US library, the solar metallicity stars and the stars in common between Indo-US and the MILES library, respectively. The median $F W H M$ of the MILES-based stellar population models is $2.31 \pm 0.11 \AA$.

\subsection{Template: $M A R C S$ library at $R=20000$}

We use all solar metallicity MILES stars and combine them with the theoretical MARCS library selecting the spectra at intervals 
of $T=500 \mathrm{~K}$ (92 spectra in total). We made a test using the whole MARCS library (342 stars) instead of this subsample and the results were consistent, suggesting that we are not affected by template mismatch. We followed the same procedure as above and we found the same issue for the latest and first wavelength bin in both the stellar spectra and integrated models. The results are shown in panel b) of Fig. 2. The median FWHM is $2.55 \pm$ $0.14 \AA$ and $2.57 \pm 0.13 \AA$ for stars and stellar population models, respectively.

\subsection{Template: ELODIE v3.1 library at $R=42000$}

We select MILES and ELODIE solar metallicity templates (653 spectra) with the same criteria as for the Indo-US library. Since ELODIE covers a shorter wavelength range (3900-6800 ^) than MILES (3525-7500 A) we select the MILES spectra in the ELODIE region. We divided the spectra in 8 wavelength regions instead of 11 for roughly matching the bins we used in the two tests described above. We also tested the line broadening derived by fitting the 303 stars in common between MILES and ELODIE, by fitting each star with the equivalent in the other library. Note that even though the use of ELODIE limits our test to a shorter wavelength range, we are still able to cover the most important spectral features for extracting the stellar kinematics. The results are shown in panel c) of Fig. 2.

The median $F W H M$ we derived by using ELODIE v3.1 is $2.53 \pm 0.05 \AA, 2.55 \pm 0.09 \AA$ and $2.54 \pm 0.07 \AA$ for solar metallicity stars, stars in common between the two libraries and stellar population models, respectively.

The residuals of the fits based on the MARCS spectra are slightly larger than the one derived with ELODIE, but consistent with each other within $1 \sigma$ error. Therefore they do not affect our estimation of the line broadening. This difference in the residuals is mainly due to the typical shortcoming of theoretical libraries listed above. And yet, the results obtained with MARCS and ELODIE are fully consistent.

\subsection{Comparison and discussion}

The results we obtained with both the ELODIE and MARCS templates agree well with each other and indicate a resolution of $\sim 2.54 \pm 0.05 \AA F W H M$ for both MILES stars $^{2}$ and the stellar population models based on those stars.

This value is coarser than the nominal value for the MILES instrumental $F W H M$ of $2.3 \AA$. The resolution obtained by means of the Indo-US library, instead, is consistent with the latter. This implies that the different resolution for MILES found by Sánchez-Blázquez et al. (2006) comes from the different resolution of the underlying template - Indo-US - that was used to derive the resolution. The triple test presented here suggests that the library Indo-US has a coarser spectral resolution than previously thought.

In order to test this conclusion we re-evaluated the spectral resolution of the Indo-US library following the same procedure as described above. We fitted the solar metallicity Indo-US stars with the two high-resolution libraries MARCS and ELODIE. The results are shown in Fig. 3. The resulting median resolution of the Indo-US solar metallicity templates (146 stars) turns out to be $1.39 \pm 0.17 \AA F W H M$ and $1.32 \pm 0.09 \AA$ for the

\footnotetext{
2 The FWHM of MILES stars we found is consistent with the $\sim 2.56 \AA$ recently reported by Prugniel et al. (2011).
}
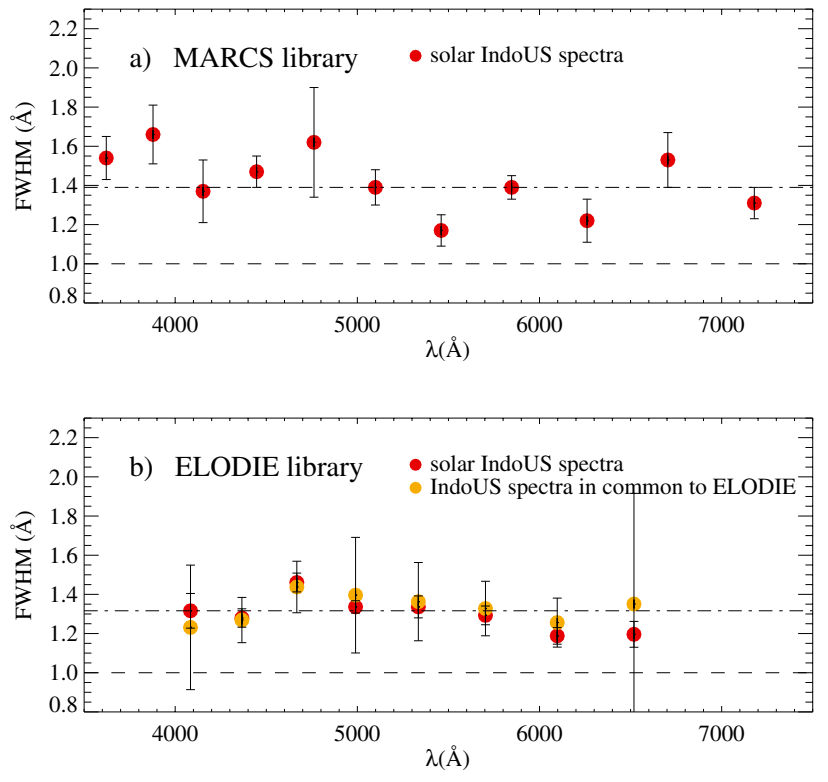

Fig. 3. $F W H M$ of Indo-US solar metallicity stars as a function of wavelength for two different templates panel a) MARCS, and panel b) ELODIE v.3.1 solar metallicity stars (red dots) and stars in common between Indo-US and ELODIE (orange dots). In each plot the dashed line represents the nominal $F W H M=1 \AA$ from Valdes et al. (2004), whereas the dotted-dashed line represents the median $F W H M$ found here.

MARCS and ELODIE templates, respectively ${ }^{3}$. This is significantly coarser than the $\sim 1.2 \AA F W H M$ value specified in Valdes et al. (2004). We conclude that the spectral resolution derived by Sánchez-Blázquez et al. (2006) for MILES was too high, mainly because the spectral resolution of the library used for the fit was overestimated. The true spectral resolutions of the MILES and Indo-US libraries are $2.54 \pm 0.05 \AA F W H M$ and $1.35 \pm 0.07 \AA$ $F W H M$, respectively.

\section{Conclusions}

Over the last few years, empirical stellar libraries have been extensively used to build stellar population models in combination with theoretical spectra. A very accurate knowledge of the spectral resolution is fundamental for avoiding propagation errors and wrong estimation of the intrinsic stellar velocity dispersion of galaxies.

In this research note we report results from tests aimed at verifying the spectral resolution of MILES library and the SSP models based on them. This exercise was done because in Maraston \& Strömbäck (2011) and Thomas et al. (2011) it was found that the spectral resolution of stellar population models based on MILES appeared to be somewhat coarser than generally assumed.

We base our test on the comparison of MILES stars with three different sets of templates, one theoretical, from the highresolution MARCS library, and two empirical, the Indo-US library initially used for assessing the spectral resolution of MILES by (Sánchez-Blázquez et al. 2006) and ELODIE v3.1. The key to our analysis is that the theoretical template has a very high and well-defined resolution, implying that any error on this

\footnotetext{
3 Considering the 300 stars in common between ELODIE and Indo-US library we find the consistent results of $1.35 \pm 0.08 \AA$.
} 
theoretical value would not affect our conclusion. The MARCS library was crucial for constraining the values of the resolution also for the other two empirical templates.

The results we obtained with both ELODIE and MARCS templates agree very well with each other and indicate a resolution of $\sim 2.54 \pm 0.05 \AA F W H M$ for both the MILES stars and the stellar population models based on those stars. This is somewhat coarser than the nominal value for the MILES resolution of $2.3 \AA$ $F W H M$. We found that the difference is due to the uncertainties on the Indo-US library FWHM. By applying the same method and using ELODIE and MARCS as templates for Indo-US, we find a resulting median resolution of $1.35 \pm 0.07 \AA F W H M$. This implies a lower spectral resolution than the $\sim 1.2 \AA F W H M$ specified in Valdes et al. (2004), which has propagated into the derivation of the MILES spectral resolution.

Acknowledgements. We acknowledge the anonymous referee for his/her many comments that improved our manuscript. We are grateful to Harald Kuntschner, Patricia Sánchez-Blázquez, Jesús Falcón-Barroso, Janine Pforr and Oliver Steele, for useful discussions. A.B., C.M. and J.J. acknowledge support by the Marie Curie Excellence Team Grant UniMass (PI C. Maraston) MEXT-CT2006-042754 of the Training and Mobility of Researchers programme financed by the European Community.

\section{References}

Baranne, A., Queloz, D., Mayor, M., et al. 1996, A\&AS, 119, 373 Bruzual, A. G., \& Charlot, S. 1993, ApJ, 405, 538 Bruzual, G., \& Charlot, S. 2003, MNRAS, 344, 1000 Cappellari, M., \& Emsellem, E. 2004, PASP, 116, 138 Fioc, M., \& Rocca-Volmerange, B. 1997, A\&A, 326, 950
Gustafsson, B., Edvardsson, B., Eriksson, K., et al. 2008, A\&A, 486, 951 Johansson, J., Thomas, D., \& Maraston, C. 2010, MNRAS, 406, 165 Kauffmann, G., Heckman, T. M., White, S. D. M., et al. 2003, MNRAS, 341, 33 Koleva, M., Prugniel, P., Ocvirk, P., Le Borgne, D., \& Soubiran, C. 2008, MNRAS, 385, 1998

Koleva, M., Prugniel, P., Bouchard, A., \& Wu, Y. 2009, A\&A, 501, 1269

Korn, A. J., Maraston, C., \& Thomas, D. 2005, A\&A, 438, 685

Kurucz, R. L. 1979, ApJS, 40, 1

Le Borgne, J., Bruzual, G., Pelló, R., et al. 2003, A\&A, 402, 433

Le Borgne, D., Rocca-Volmerange, B., Prugniel, P., et al. 2004, A\&A, 425, 881

MacArthur, L. A., González, J. J., \& Courteau, S. 2009, MNRAS, 395, 28

Maraston, C. 1998, MNRAS, 300, 872

Maraston, C. 2005, MNRAS, 362, 799

Maraston, C., \& Strömbäck, G. 2011, MNRAS, submitted

Maraston, C., Nieves Colmenárez, L., Bender, R., \& Thomas, D. 2009a, A\&A 493, 425

Maraston, C., Strömbäck, G., Thomas, D., Wake, D. A., \& Nichol, R. C. 2009b, MNRAS, 394, L107

Prugniel, P., \& Soubiran, C. 2001, A\&A, 369, 1048

Prugniel, P., Soubiran, C., Koleva, M., \& Le Borgne, D. 2007 [arXiv:astro-ph/0703658]

Prugniel, P., Vauglin, I., \& Koleva, M. 2011, A\&A, accepted [arXiv:astro-ph/1104.4952]

Sánchez-Blázquez, P., Peletier, R. F., Jiménez-Vicente, J., et al. 2006, MNRAS, 371,703

Schiavon, R. P. 2007, ApJS, 171, 146

Thomas, D., Maraston, C., \& Johansson, J. 2011, MNRAS, 412, 2183

Tremonti, C. A., Heckman, T. M., Kauffmann, G., et al. 2004, ApJ, 613, 898

Valdes, F., Gupta, R., Rose, J. A., Singh, H. P., \& Bell, D. J. 2004, ApJS, 152, 251

Vazdekis, A. 1999, ApJ, 513, 224

Vazdekis, A., Sánchez-Blázquez, P., Falcón-Barroso, J., et al. 2010, MNRAS, 404, 1639

Worthey, G. 1994, ApJS, 95, 107

Wu, Y., Singh, H. P., Prugniel, P., Gupta, R., \& Koleva, M. 2011, A\&A, 525, A71

York, D. G., Adelman, J., Anderson, Jr., J. E., et al. 2000, AJ, 120, 1579 